\title{
Smart Artificial Intelligence Computerized Models for Shelf Life Prediction of Processed Cheese
}

\author{
Sumit Goyal, Gyanendra Kumar Goyal \\ National Dairy Research Institute, Karnal -132001, India \\ E-mail: thesumitgoyal@gmail.com; gkg5878@yahoo.com
}

\begin{abstract}
Linear Layer (Design) and multiple linear regression artificial intelligence computerized models were developed for predicting shelf life of processed cheese stored at $7-8^{\circ} \mathrm{C}$. Mean Square Error, Root Mean Square Error, Coefficient of Determination and Nash - Sutcliffo Coefficient were applied for comparing the prediction ability of the developed models. The modelling results showed excellent agreement between the experimental data and predicted values with a high determination coefficient, suggesting that the Linear Layer (Design) and MLR models are very efficient in predicting the shelf life of processed cheese stored at 7-8oC.
\end{abstract}

Keywords: artificial intelligence, artificial neural network, linear layer (design), multiple linear regression, processed cheese, prediction

\section{Introduction}

\subsection{Artificial Neural Network (ANN)}

ANN is usually called neural network is a mathematical model or computational model that is inspired by the structure and functional aspects of ANN. ANN based computing method is an adaptive system that changes its structure based on external or internal information that flows through the network during the learning phase. In ANN based intelligent computing, simple artificial nodes called "neurons", "neurodes", "processing elements" or "units" are connected together to form a network of nodes mimicking the biological neural networks - hence the 
term "ANN". Generally, ANN involves a network of simple processing elements that exhibit complex global behavior determined by connections between processing elements and element parameters. While an ANN does not have to be adaptive, its practical use comes with algorithms designed to alter the weights of the connections in the network to produce a desired signal flow [1].

\subsection{Linear Layer (Design)}

Linear layers are single layers of linear neurons. They may be static, with input delays of 0 , or dynamic, with input delays greater than 0 . They can be trained on simple linear time series problems, but often are used adaptively to continue learning while deployed, so they can adjust to changes in the relationship between inputs and outputs while being used [2].

\subsection{Multiple Linear Regression (MLR)}

MLR is a technique that allows additional factors to enter the analysis separately so that the effect of each can be estimated. It is valuable for quantifying the impact of various simultaneous influences upon a single dependent variable. Further, because of omitted variables bias with simple regression, multiple regression is often essential even when the investigator is only interested in the effects of one of the independent variables [3].

\subsection{Processed Cheese}

Processed cheese occupies a prominent place in food industry and is one of the most liked dairy products. It is prepared by using grated medium ripened (up to six months old) Cheddar cheese. Often, a part of medium ripened cheese is replaced by fresh or less ripened Cheddar cheese. During its manufacture, emulsifiers, water, extra salt, preservatives, food colorings and selected spices (optional) are added. The mixture is heated to $70^{\circ} \mathrm{C}$ for $10-15$ minutes with steam in a cleaned double jacketed stainless steel kettle, which is open, shallow and round-bottomed, with continuous gentle stirring (about 50-60 circular motions per minute) with a flattened ladle in order to get unique body \& texture in the final product. Today, several varieties of processed cheese with different flavours, colors, and textures are available in the world market. Processed cheese has many advantages over unprocessed cheese, viz., pleasing taste, extended shelf life, and the use of emulsifiers in processed cheese preparation results in cheese that melts smoothly when heated. Processed cheeses are normally very smooth, mediumfirm, velvety, and highly regarded by cheese lovers throughout the world. 


\subsection{Shelf Life}

Martins et al. [4] defined shelf life as the length of time that a product is acceptable and meets the consumer's expectations regarding food quality. It is the result of the conjunction of all services in production, distribution, and consumption. Shelf life dating is one of the most difficult tasks in food engineering. Market pressure has led to the implementation of shelf life by sensory analyses, which may not reflect the full quality spectra. Moreover, traditional methods for shelf life dating and small-scale distribution chain tests cannot reproduce in a laboratory the real conditions of storage, distribution, and consumption on food quality.

\subsection{Shelf Prediction Using ANNs}

Goyal and Goyal [5] implemented brain based artificially intelligent scientific computing models for shelf life detection of cakes stored at $30 \mathrm{oC}$. The potential of simulated neural networks for predicting shelf life of soft cakes stored at $10 \mathrm{oC}$ was highlighted by Goyal and Goyal [6]. Cascade single and double hidden layer models were developed and compared with each other for predicting the shelf life of Kalakand, a desiccated sweetened dairy product [7]. For forecasting the shelf life of instant coffee drink, radial basis artificial neural engineering and multiple linear regression models were suggested [8]. Cascade forward and feedforward backpropagation artificial intelligence models for prediction of sensory quality of instant coffee flavoured sterilized drink have been evolved [9-10]. ANN for predicting the shelf life of milky white dessert jeweled with pistachio was applied by Goyal and Goyal [11]. The shelf life of brown milk cakes decorated with almonds was predicted by developing artificial neural network based radial basis (exact fit) and radial basis (fewer neurons) models [12]. Also, the time-delay and linear layer (design) intelligent computing expert system models have been developed for predicting the shelf life of soft mouth melting milk cakes stored at 6oC [13]. Computerized models predicted shelf life of post-harvest coffee sterilized milk drink [14].

This present study aims to develop linear layer (design) and multiple linear regression computerized models for predicting the shelf life of processed cheese stored at $7-8^{\circ} \mathrm{C}$.

\section{Method Material}

Experimentally developed 36 observations for each input and output variables were used for developing the models. The dataset was randomly divided into two disjoint subsets, namely, training set having 30 (80\% for training) observations, and validation set (20\% for testing) consisting of 6 observations. 


$$
\begin{aligned}
M S E & =\left[\sum_{1}^{N}\left(\frac{Q_{\exp }-Q_{c a l}}{n}\right)^{2}\right] \\
R M S E & =\sqrt{\frac{1}{n}\left[\sum_{1}^{N}\left(\frac{Q_{\exp }-Q_{\text {cal }}}{Q_{\text {exp }}}\right)^{2}\right]} \\
R^{2} & =1-\left[\sum_{1}^{N}\left(\frac{Q_{\exp }-Q_{c a l}}{Q_{\exp }{ }^{2}}\right)^{2}\right] \\
E^{2} & =1-\left[\sum _ { 1 } ^ { N } \left(\frac{Q_{\exp }-Q_{c a l}}{\left.\left.Q_{\exp }-\overline{Q_{\text {exp }}}\right)^{2}\right]}\right.\right.
\end{aligned}
$$

Where, $Q_{\exp }=$ Observed value; $Q_{\text {cal }}=$ Predicted value; $Q_{\exp }^{-}=$Mean predicted value; $n=$ Number of observations in dataset. Mean Square Error MSE (1), Root Mean Square Error RMSE (2), Coefficient of Determination $R^{2}$ (3) and Nash - Sutcliffo Coefficient $E^{2}(4)$ were applied in order to compare the prediction ability of the developed models.

The input variables of the network were the experimental data pertaining to soluble nitrogen, $\mathrm{pH}$; standard plate count, Yeast \& mould count, and spore count. The sensory score assigned by the trained panelists was taken as output variable for developing computerized models (Figure 1). 


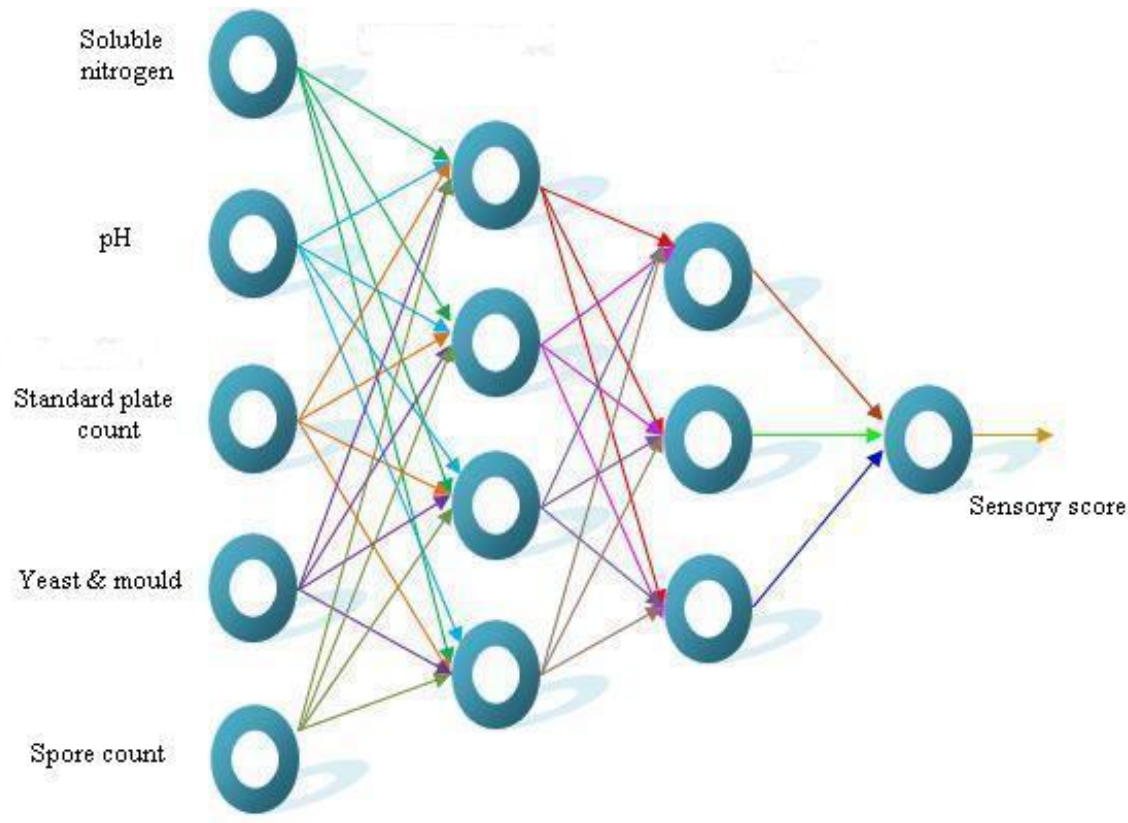

Figure 1. Input and output parameters for Linear layer (design) and MLR models

\section{Results and Discussion}

Linear Layer (Design) (Table 1) and MLR (Table 2) were developed and compared with each other for predicting shelf life of processed cheese stored at 7$8^{\circ} \mathrm{C}$.

Table 1: Results of Linear Layer (Design) model

\begin{tabular}{cccc}
\hline MSE & RMSE & $\mathbf{R}^{2}$ & $\mathbf{E}^{2}$ \\
\hline $1.22701 \mathrm{E}-05$ & 0.003502871 & 0.996497129 & 0.99998773
\end{tabular}

Table 2: Results of MLR model

\begin{tabular}{cccc}
\hline MSE & RMSE & $\mathrm{R}^{2}$ & $\mathrm{E}^{2}$ \\
\hline $5.48272 \mathrm{E}-06$ & 0.002341522 & 0.997658478 & 0.999994517
\end{tabular}




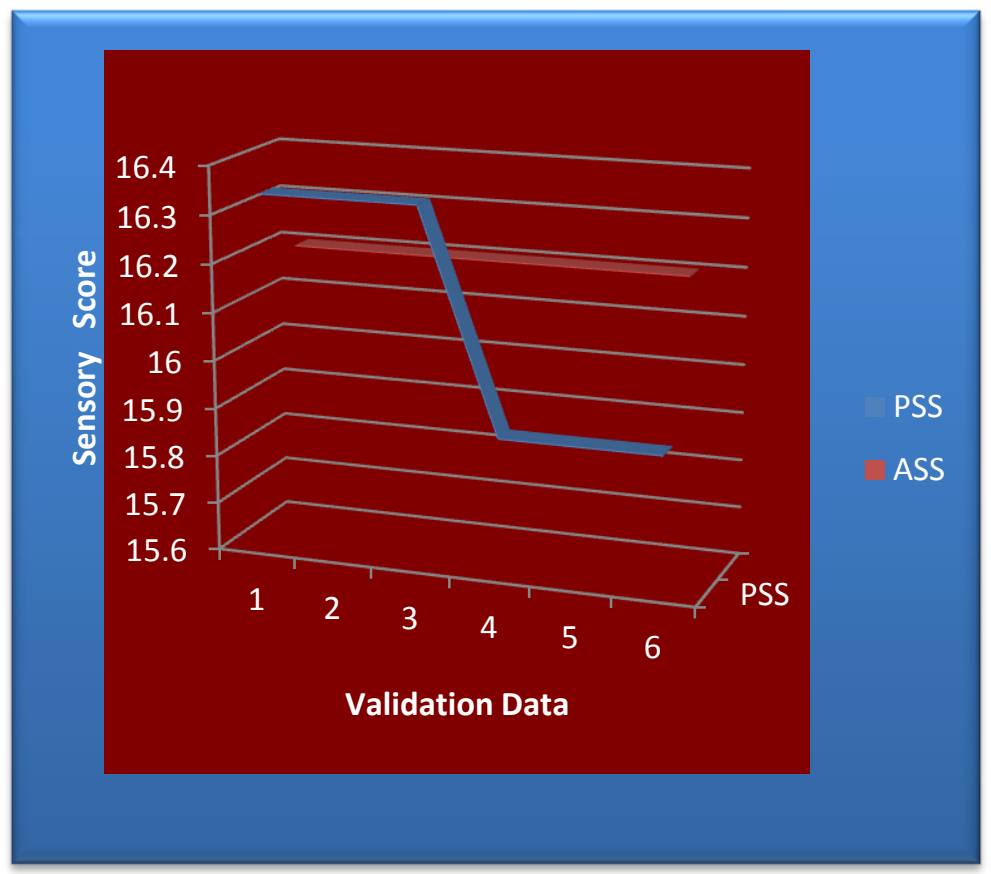

Figure 2. Comparison of ASS and PSS for Linear Layer (Design) model

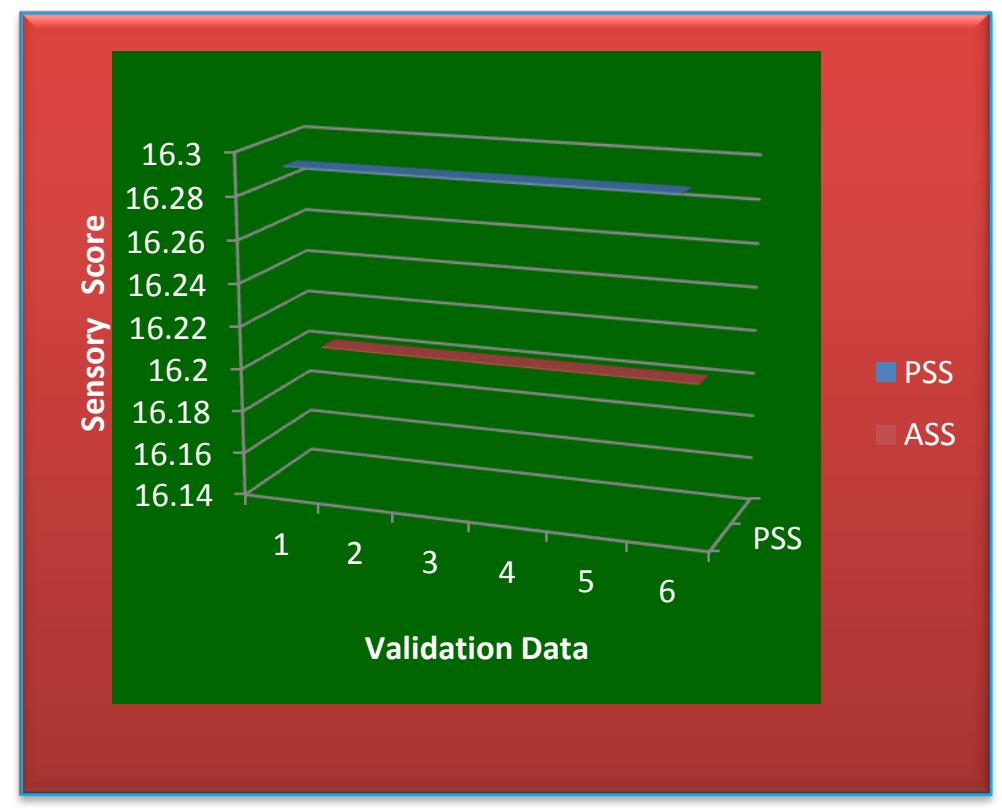

Figure 3. Comparison of ASS and PSS for MLR model

The comparison of Actual Sensory Score (ASS) and Predicted Sensory Score (PSS) for the developed computerized models are illustrated in Figure 2 and Figure 3, respectively. 
On comparing the two developed computerized models, viz., Linear Layer (Design) (MSE: 1.22701E-05,RMSE: 0.003502871, R2 : 0.996497129:,E2 : 0.99998773), and MLR (MSE: 5.48272E-06, RMSE : 0.002341522,R2: 0.997658478 ,E2 : 0.999994517), it was observed that both the models got simulated very well with less than $1 \%$ RMSE, revealing that the Linear Layer (Design) and MLR models are very efficient in predicting the shelf life of processed cheese stored at 7-8 oC.

\section{Conclusion}

For predicting the shelf life of processed cheese stored at 7-8oC, Linear Layer (Design) and Multiple Linear Regression models were developed. The input variables of the network were soluble nitrogen, $\mathrm{pH}$; standard plate count, yeast \& mould count, and spore count. The output variable was sensory score of the processed cheese. The modelling results showed that there was excellent agreement between the experimental data and the predicted values, with a high determination coefficient $(\mathrm{R} 2=0.996497129 ; 0.997658478)$ establishing that the developed models were able to analyze non-linear multivariate data with very good performance, fewer parameters, and shorter calculation time. The model might be an alternative low cost and less time consuming method to control the expiration date of processed cheese, shown on labels and provide consumers with a safer food supply [14-21].

\section{References}

[1] http://en.wikipedia.org/wiki/Artificial_neural_network (accessed on 28.1.2011).

[2] http://www.mathworks.com/help/toolbox/nnet/ref/linearlayer.html (accessed on 15.1.2011).

[3] http://www.law.uchicago.edu/files/files/20.Syks.Regression.pdf (accessed on 29.4.2011).

[4] Martins, R. C., Lopes, V.V., Vicente, A.A. \& Teixeira, J. A. (2008). Computational shelf-life dating: complex systems approaches to food quality and safety. Food and Bioprocess Technology, 1(3), 207-222.

[5] Goyal, Sumit \& Goyal, G.K. (2011). Brain based artificial neural network scientific computing models for shelf life prediction of cakes. Canadian Journal on Artificial Intelligence, Machine Learning and Pattern Recognition, 2(6), 73-77.

[6] Goyal, Sumit \& Goyal, G. K. (2011).Simulated neural network intelligent computing models for predicting shelf life of soft cakes. Global Journal of Computer Science and Technology, 11(14), version 1.0, 29-33.

[7] Goyal, Sumit \& Goyal, G.K. (2011). Advanced computing research on cascade single and double hidden layers for detecting shelf life of kalakand: 
An artificial neural network approach. International Journal of Computer Science \& Emerging Technologies, 2(5), 292-295.

[8] Goyal, Sumit \& Goyal, G.K. (2011). Application of artificial neural engineering and regression models for forecasting shelf life of instant coffee drink. International Journal of Computer Science Issues, 8(4), no.1, 320-324.

[9] Goyal, Sumit, \& Goyal, G.K. (2011). Cascade and feedforward backpropagation artificial neural networks models for prediction of sensory quality of instant coffee flavoured sterilized drink. Canadian Journal on Artificial Intelligence, Machine Learning and Pattern Recognition, 2(6), 7882.

[10] Goyal, Sumit \& Goyal, G.K. (2011).Development of neuron based artificial intelligent scientific computer engineering models for estimating shelf life of instant coffee sterilized drink. International Journal of Computational Intelligence and Information Security, 2(7), 4-12.

[11] Goyal, Sumit \& Goyal, G.K. (2011). A new scientific approach of intelligent artificial neural network engineering for predicting shelf life of milky white dessert jeweled with pistachio. International Journal of Scientific and Engineering Research, 2(9), 1-4.

[12] Goyal, Sumit \& Goyal, G.K. (2011). Radial basis artificial neural network computer engineering approach for predicting shelf life of brown milk cakes decorated with almonds. International Journal of Latest Trends in Computing, 2(3), 434-438.

[13] Goyal, Sumit, \& Goyal, G.K. (2011). Development of intelligent computing expert system models for shelf life prediction of soft mouth melting milk cakes. International Journal of Computer Applications, 25(9), 41-44.

[14] Goyal, Sumit and Goyal, G.K. (2011). Computerized models for shelf life prediction of post-harvest coffee sterilized milk drink. Libyan Agriculture Research Center Journal International, 2 (6), 274-278.

[15] Goyal, Sumit and Goyal, G.K. (2012). Radial basis (exact fit) and linear layer (design) computerized ANN models for predicting shelf life of processed cheese. Computer Science Journal, 2(1), 11-18.

[16] Goyal, Sumit and Goyal, G.K. (2012). Soft computing methodology for shelf life prediction of processed cheese. International Journal of Informatics and Communication Technology, 1(1), 1-5.

[17] Goyal, Sumit and Goyal, G.K. (2012). Predicting shelf life of burfi through soft computing. International Journal of Information Engineering and Electronic Business, 4(3), 26-33.

[18] Goyal, Sumit and Goyal, G.K. (2012). Predicting shelf life of dairy product by using artificial neural networks (ANN) and statistical computerized methods. International Journal of Computer Engineering Research, 3(2), 2024.

[19] Goyal, Sumit and Goyal, G.K. (2012). Artificial Neural Networks for Dairy Industry: A Review. Journal of Advanced Computer Science \& Technology, 1(3), 101-115. 
[20] Goyal, Sumit and Goyal, G.K. (2012). Estimating processed cheese shelf life with artificial neural networks. International Journal of Artificial Intelligence, 1(1), 19-24.

[21] Goyal, Sumit and Goyal, G.K. (2012). Time-Delay artificial neural network computing models for predicting shelf life of processed cheese. BRAIN. Broad Research in Artificial Intelligence and Neuroscience, 3(1), 63-70. 